\title{
Influence of Inlet Duct Length on the Hydraulic Performance of the Waterjet Propulsion Device
}

\author{
Xionghuan Chen, Li Cheng ${ }^{(D}$, Chuan Wang $\mathbb{D}$, and Can Luo \\ College of Hydraulic Science and Engineering, Yangzhou University, Yangzhou 225009, China \\ Correspondence should be addressed to Li Cheng; chengli@yzu.edu.cn and Chuan Wang; wangchuan198710@126.com
}

Received 16 December 2020; Revised 4 March 2021; Accepted 12 May 2021; Published 29 May 2021

Academic Editor: Peter Mucka

Copyright (c) 2021 Xionghuan Chen et al. This is an open access article distributed under the Creative Commons Attribution License, which permits unrestricted use, distribution, and reproduction in any medium, provided the original work is properly cited.

\begin{abstract}
This study uses a combination of computational fluid dynamics (CFD) and experimental research to explore the influence of the length of the inlet duct on the hydraulic performance of the water jet propulsion pump device. By extending the oblique, straight, and long pipe section of the inlet duct, six sets of schemes were designed. The research results show that as the length of the inlet duct increases, the head, thrust, and inlet duct efficiency of the water jet propulsion pump first increase and then decrease, reaching the maximum value in scheme 4 , but it has little effect on the efficiency of the entire water jet propulsion system. In addition, after extending the obliquely straight long pipe section of the inlet flow channel, the velocity distribution of the outlet section of the flow channel is gradually uniform, and the absolute value of the vorticity first increases and decreases, but the uniformity of the velocity distribution and the weighted average angle change little. This research enriches the research theory of the inlet duct and also provides a reference for the selection of inlet duct length and its performance optimization.
\end{abstract}

\section{Introduction}

Although traditional propeller propulsion has high propulsion efficiency in low and medium speeds, and the propeller propulsion efficiency of displacement ships is about $65 \%$ [1], the water jet propulsion pump device has high efficiency and low noise when the ship is traveling at high speed, and it is widely used on high-speed ships [2]. In the past decades, with the rapid development of computer technology, CFD technology has been widely used in many fields, among which a lot of research has been done on the hydraulic performance of pumps in fluid machinery [3-7]. In order to ensure the accuracy of numerical simulation, a large number of studies through the combination of experimental and numerical simulation methods were compared to obtain more reliable research results [8-12]. All kinds of mathematical methods have been widely used, combined with numerical simulation to solve the practical engineering problems [13-16]. The thrust of water jet propulsion is obtained by the reaction force of the water jet from the propulsion pump. One of the main components of the water inlet duct is to transfer the water flow from the bottom of the ship to the propulsion pump. Therefore, the performance of the water inlet duct will affect the performance of the entire propulsion system.

Regarding the parameter optimization of the inlet duct, Jiao et al. $[17,18]$ analyzed the relationship between the performance of the inlet duct and the ship's speed and obtained the geometric parameter values under the best optimized condition. Huang et al. [19] improved the NSGA-II and TOPSIS technologies and optimized the performance of the inlet duct with the unevenness and verticality of the outlet and hydraulic efficiency as the optimization goals. Gong et al. [20] designed a model predictive controller for steering system control. Regarding the research on the internal flow characteristics of the inlet duct, Park et al. [21] analyzed the velocity vector and streamline numerical results of the inlet duct as well as the pressure distribution, showed the eddy caused by the separation along the corner of the side wall, and explained the flow phenomenon inside the inlet channel. Ding and Wang [22] used computational fluid dynamics 
to determine the flow loss of the inlet channel, thereby further improving the prediction accuracy of the propulsion performance of the entire water jet propulsion system. Xu et al. [23] used HSV to show the evolution of TLC and solved the problem of tip leakage cavitation. Cao et al. [24] carried out numerical simulations on the water jet propulsion pump device and revealed the internal relationship between the unevenness of the internal flow of the inlet duct and the performance deviation of the water jet propulsion pump device and essentially studied the performance deviation between the uniform and nonuniform suction flow. Regarding the innovation of the research method of the inlet flow path, Huang and Luo [25] improved the NSGA-II and TOPSIS technologies and optimized the performance of the inlet flow channel with the unevenness and verticality of the outlet and hydraulic efficiency as the optimization goals. Lindau et al. [26] used the RANS method to conduct an in-depth study on the cavitation performance of water jet axial flow pumps. Gong et al. [27] used PIV to measure the velocity distribution. Kimball and Taylor [28], Kerwin [29], etc., based on the lifting surface theory of propeller design and combined with RANS numerical calculations, carried out research on the design method of water jet propulsion pumps and obtained certain law. Gong et al. [30] studied the interaction between the water jet propulsion system and the hull and found that the different design of the inlet passage would lead to different interactions between them, leading to the performance differences among the four water jet propulsion systems. Predecessors have carried out a lot of research work on the inlet duct and the entire water jet propulsion pump device, but there are few aspects of the analysis and optimization of the inlet duct parameters.

In view of this, the performance optimization of the inlet duct is of great significance to the application of the entire water jet propulsion pump device. In order to understand the influence of the length of the inlet duct on the external characteristics of the inlet duct, the uniformity of the outlet of the inlet duct, and the internal characteristics of the inlet duct, numerical simulation and qualitative and quantitative analyses will be carried out in this article. This is conducive to the application of the water jet propulsion pump device on ships and also expands the theoretical analysis content of the inlet duct.

\section{Numerical Simulation Methods}

2.1. ComputationalDomain. Because the performance of the inlet channel is inseparable from the hull structure and working conditions, and the performance of the inlet channel will also affect the hydraulic performance of the propulsion pump, the control body should be around the propulsion pump and the water inlet at the bottom of the stern when performing performance analysis on the inlet duct. Figure 1 shows the entire water jet propulsion system, which includes the inlet duct, propulsion pump (mixed flow pump), and nozzles. According to the literature [31], the length, width, and height of the flow field control body are, respectively, $30 D, 10 D$, and $8 D(D$ is the diameter of the outlet of the inlet duct, $D=223.4 \mathrm{~mm}$ ).

2.2. Mesh Generation and Independence Analysis. Use ICEM software to complete the structured grid of the inlet duct, the propulsion pump, and the water body; the schemes with different grid numbers are shown in Table 1 . As the number of grids increases, the accuracy of simulation results gradually improves, but at the same time the demand for computing resources also increases. Domestic and foreign institutions have made clear guidance and definitions for CFD credibility analysis, and most of them use Richardson extrapolation to evaluate errors caused by the calculation domain. Whether it is a high-order or low-order format, the numerical calculation results of the grid will tend to be close to the exact solution [32].

In order to obtain the most suitable number of grids, the method of changing the grid size of the water inlet channel is used to verify the grid independence; that is, as the number of grids increases, the efficiency of the water jet propulsion pump device is calculated until the calculation result is within the allowable range.

As shown in Figure 2, when the grid number of the inlet flow channel is 1.26 million, 1.35 million, and 1.48 million, the efficiency of the pump device hardly changes. The calculation results show that when the number of grids exceeds 1.35 million, the change of pump device efficiency is $0.018 \%$ and $0.004 \%$, respectively, which can be regarded as the result has nothing to do with the grid. Therefore, the number of grids of the inlet runner is 1.35 million (as shown in Figure 3).

2.3. Boundary Conditions. This article is a steady calculation. IVR $=0.45$ (IVR is the ratio of the ship speed to the averaged axial outflow velocity at the passage outlet). In this paper, the inlet boundary of the water body is set as the speed inlet, and the speed is $8 \mathrm{~m} / \mathrm{s}$. The nominal turbulence intensity (value 5\%) is used, and the hydraulic diameter is $993 \mathrm{~mm}$. Both the outlet of the water body and the outlet of the nozzle are set to average static pressure, the value is one atmospheric pressure, and the impeller speed is $700 \mathrm{r} / \mathrm{min}$. The frame change/mixing model of the two interfaces related to the impeller is set to frozen rotor. The frame change/mixing model of the other interfaces is set to frozen rotor. The turbulence model selects the standard $k-\varepsilon$ turbulence model [33], applies the first-order upwind scheme, and sets the convergence accuracy to $10^{-5}$ (as shown in Figure 4).

2.4. Research Case. In order to analyze the influence of the length of the inlet duct on itself and the entire water jet propulsion system, six schemes were designed (as shown in Table 2). Taking option 4 as the original schemes, the length of the inclined straight pipe section is $L=1.12 D$ ( $D$ is the diameter of the outlet section of the inlet duct), the inclination angle of the inlet duct is $35^{\circ}$, and the inlet duct height $H=L \cos 35^{\circ}+h$ ( $h$ is the vertical distance between the upper 


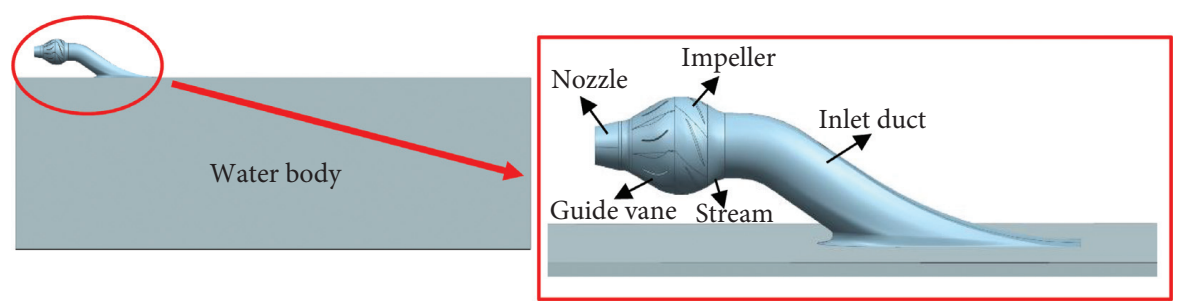

Figure 1: Computational domain.

TABLE 1: Different grid numbers of inlet duct. Unit: ten thousand.

\begin{tabular}{lccccc}
\hline Mesh & Inlet duct & Guide vane & Impeller & Nozzle & Total \\
\hline 1 & 114 & 55 & 97 & 58 & 324 \\
2 & 126 & 55 & 97 & 58 & 336 \\
3 & 135 & 55 & 97 & 58 & 345 \\
4 & 148 & 55 & 97 & 38 & 35 \\
5 & 158 & 55 & 97 & 58 & 368 \\
\hline
\end{tabular}

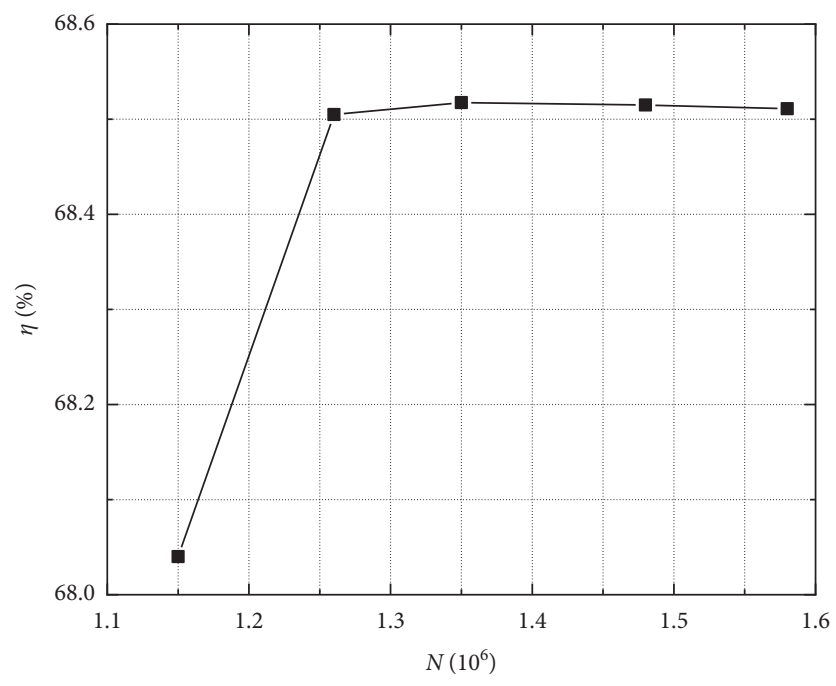

Figure 2: Hydraulic loss for different grid numbers.

end of the inclined straight pipe section and the lower end of the inlet duct exit section) (as shown in Figure 5). When the length $L$ of the inclined straight pipe section is changed, the length of the entire inlet duct will change accordingly. Because the angle is constant, the height of the runner also changes accordingly (as shown in Table 2). Through the analysis of these six schemes, certain rules can be drawn.

\subsection{Analysis Parameters}

2.5.1. External Characteristic. Efficiency is a comprehensive performance index for evaluating water jet propulsion system, which can well reflect the pros and cons of water jet propulsion system. Therefore, formulas (1)-(3) and formula (4) will be used to calculate the inlet duct efficiency $\eta_{d}$ and the efficiency $\eta_{s}$ of the entire system, respectively, and $\eta-$ $\eta_{0} / \eta_{0}$ will be used as the analysis index to obtain the graph of the change rule of efficiency with the length of the inlet duct, where $\eta_{0}$ represents the efficiency of the inlet runner in the fourth scheme.

The formula for calculating the efficiency of the inlet duct is

$$
\begin{gathered}
\eta_{d}=\frac{E_{\text {out }}}{E_{\text {in }}} \cdot 100 \%, \\
E_{\text {in }}=\frac{1}{2} \rho v_{\text {in }}^{2}+P_{\text {in }}, \\
E_{\text {out }}=\frac{1}{2} \rho v_{\text {out }}^{2}+P_{\text {out }} .
\end{gathered}
$$

In the formula, $\eta_{d}, E_{\text {in }}$, and $E_{\text {out }}$ are the inlet duct efficiency, the total energy of the runner inlet, and the total energy of the runner exit, respectively.

Water Jet Propulsion System Efficiency. The physical meaning of system propulsion efficiency is the ratio of the 


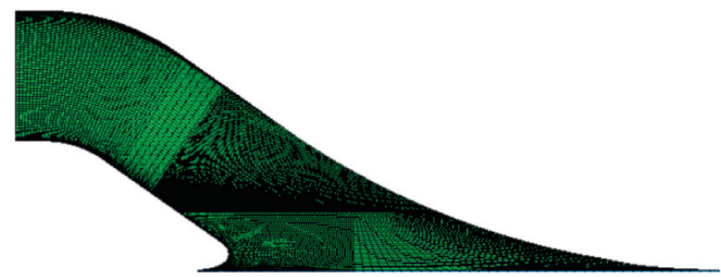

FIGURE 3: Inlet duct structured grid.

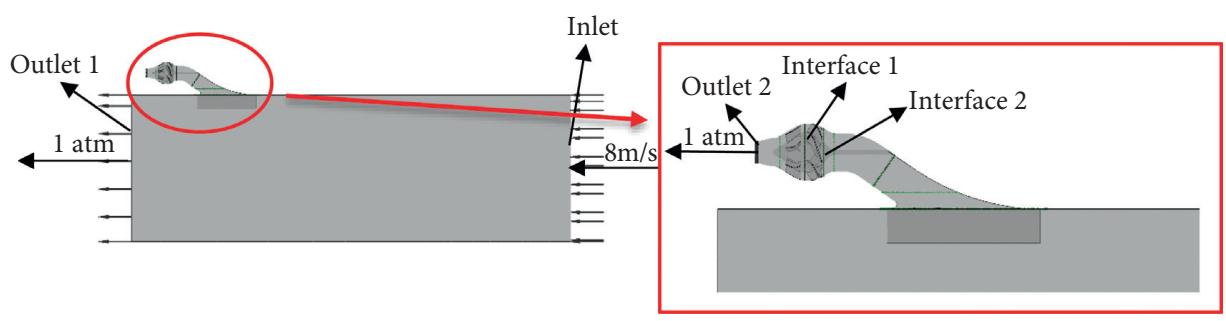

Figure 4: Schematic diagram of boundary conditions (interface 1: between impeller and guide vane; interface 2: between impeller and stream).

output power of the system to the output power of the propulsion pump [34], and considering the pipeline loss coefficient $K_{1}$, since $\mathrm{Q}=A v_{\text {out }}$ ( $A$ is the nozzle area), it is brought into

$$
\eta_{s}=\frac{2\left(\left(v_{\text {out }} / v_{s}\right)-\alpha\right)}{\left(v_{\text {out }} / v_{s}\right)^{2}-1+K_{1}} .
$$

In the formula, $v_{\text {out }}$ is the axial flow velocity of the nozzle; $v_{\mathrm{s}}$ is the speed of the ship; $\alpha$ is the influence coefficient of the boundary layer, generally 0.95 ; and $K_{1}$ is the pipeline coefficient, generally 0.45 .

2.5.2. Analysis of Flow Uniformity. In order to quantitatively analyze the flow uniformity of the outlet part of the inlet duct (as shown in Figure 6), the objective function velocity distribution uniformity $V_{u}$ and the weighted average angle $\theta$ are hereby selected as the judgment basis. ( $\theta$ refers to the weighted average of the speed of each node on the selected section).

Speed distribution uniformity is represented as

$$
V_{u}=\left[1-\frac{1}{u_{a}} \sqrt{\frac{\sum\left(u_{a i}-u_{a}\right)^{2}}{m}}\right] \times 100 \%,
$$

where $V_{u}$ is the flow velocity distribution uniformity of the outlet section of the inlet duct; $u_{a}$ is the average axial velocity of the inlet section of the impeller chamber; $u_{a i}$ is the axial velocity of each unit at the inlet section of the impeller chamber; and $m$ is the number of units divided by the section during numerical calculation of the flow field. is

Weighted average angle of exit velocity distribution [35]

$$
\theta=\frac{\sum u_{a i}\left(90^{\circ}-\tan ^{-1}\left(u_{t i} / u_{a i}\right)\right)}{\sum u_{a i}}
$$

where $\theta$ is the weighted average angle of velocity at the exit section of the runner; $u_{a i}$ is the axial velocity of each unit at the inlet section of the impeller chamber; and $u_{t i}$ is the lateral velocity of each unit at the inlet section of the pump.

2.5.3. Thrust Characteristics. The water jet propulsion system relies on the water jet from the nozzle to obtain the reverse thrust to propel the ship. Therefore, the thrust of the water jet propulsion system is a very important indicator. In an ideal state, the outlet flow from the nozzle should not rotate at all, only the axial high-speed flow part, so the velocity distribution of the nozzle is closely related to the thrust of the water jet [36]. Taking the computational domain in this article as the controlling body, according to the momentum theorem, we can get

$$
\begin{gathered}
Q_{\text {in }}=Q_{\text {out }}, \\
Q_{\text {in }}=A_{\text {in }} v_{\text {in }}, \\
Q_{\text {out }}=A_{\text {out }} v_{\text {out }} .
\end{gathered}
$$

To this end, the following formula is used to calculate the thrust of the device, and the relative increment is calculated on the basis of Scheme 4 .

Thrust of the whole device [37] is

$$
\begin{aligned}
& F_{T}=\rho Q v_{\text {out }}-v_{\text {in }}, \\
& v_{\text {in }}=\alpha v_{s}, \\
& Q=A V_{\text {out }},
\end{aligned}
$$

where $A$ is the cross-sectional area of the nozzle; $\rho$ is the fluid density; $v_{\text {out }}$ is the axial flow velocity of the nozzle; $v_{\text {in }}$ is the flow velocity at the inlet of the inlet duct; $v_{s}$ is the speed of the ship; and $\alpha$ is the boundary layer influence coefficient, generally 0.95 . 
TABLE 2: Research cases.

Case number

Case 1

Case 2

Case 3

Case 4

Case 5

Case 6

$1.4 L$
Diagram
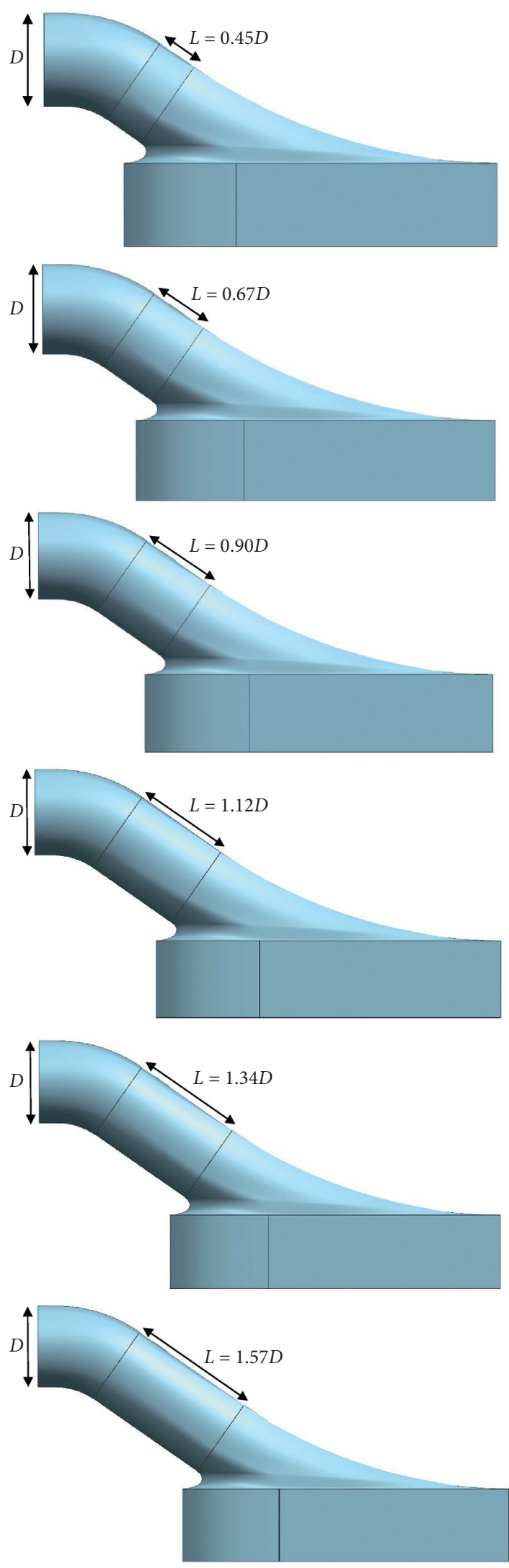


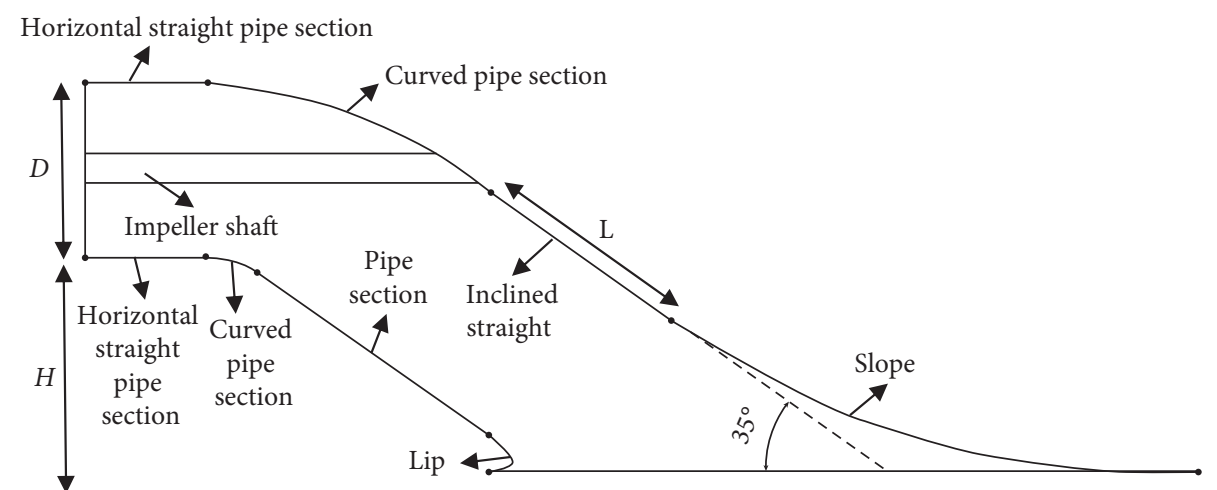

FIgURE 5: Schematic diagram of the geometric structure of the inlet duct.

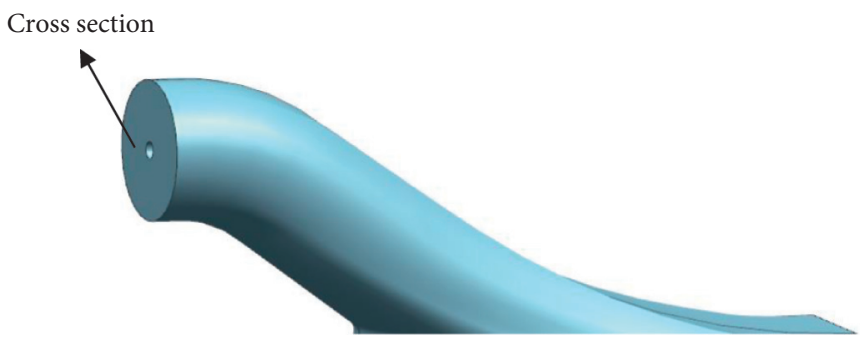

Figure 6: Schematic diagram of cross section.

\section{Experimental Verification}

In this paper, the model in the literature [38] is selected. In order to verify the reliability of the numerical simulation, the same boundary conditions as in the literature are set to calculate the efficiency of the water jet propulsion pump under the same working conditions. The selected test equipment is shown in Figure 7. A test facility is built which consists of two closed-loop circuits. One circuit is used to provide navigation speed for the propulsion pumping system. The other is applied to measuring performance of the pumping system. The propulsion pumping system consists of a propulsion pump, a guide vane, and an inlet passage. The pump is driven by a DC electric motor via a system. A frequency controller is used to regulate the shaft speed. The flow rate of the pump is measured by an electromagnetic flow meter with an accuracy of $\pm 0.5 \%$. The system uncertainty of the instrument measuring the head is $\pm 0.1 \%$ (the uncertainty of the entire experiment includes system uncertainty and random uncertainty).

Figure 8 is a performance comparison between test results and experimental results. The graph uses a series of flow points as the abscissa and head and efficiency as the ordinate to compare the numerical simulation results with the experimental data. It can be seen from the figure that the head decreases with the increase of the flow, and the efficiency increases with the increase of the flow. The experimental results of the water jet propulsion device and the overall trend of the numerical results are consistent, so the numerical simulation results in this article are credible.

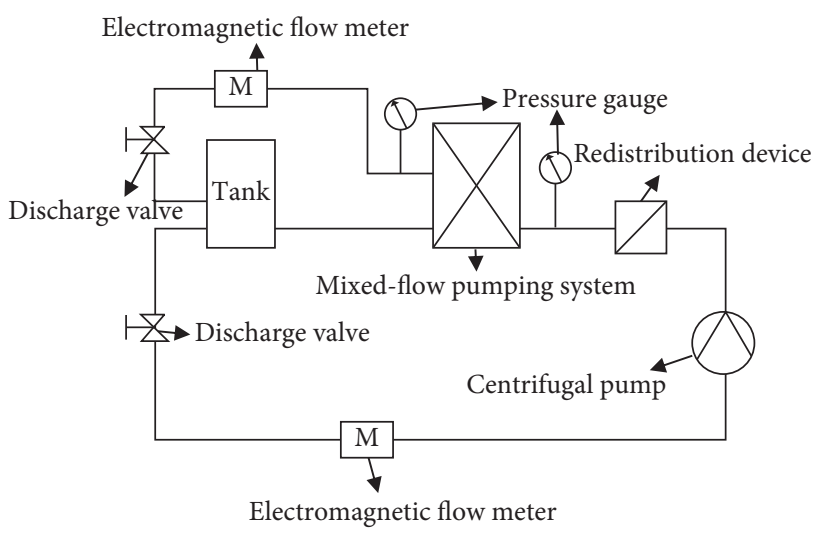

Figure 7: Waterjet propulsion device test system [38].

\section{Performance Analysis}

In order to fully analyze the influence of the length of the inlet duct on the inlet duct and the entire water jet propulsion system, the following will present a quantitative and qualitative analysis from three aspects: the external characteristics of the inlet duct, the uniformity of the outlet flow, and the internal flow of the runner. A certain rule is found to facilitate the application of runners at different lengths.

4.1. External Characteristic Analysis. Figure 9 shows the variation of the head $H$ and system efficiency $\eta_{s}$ with the length of the inlet flow channel. $H_{0}$ and $\eta_{s 0}$ are the head and system efficiency of the original scheme. It can be seen from the figure that the efficiency of the system does not change much with the length of the inlet duct, and the variation 


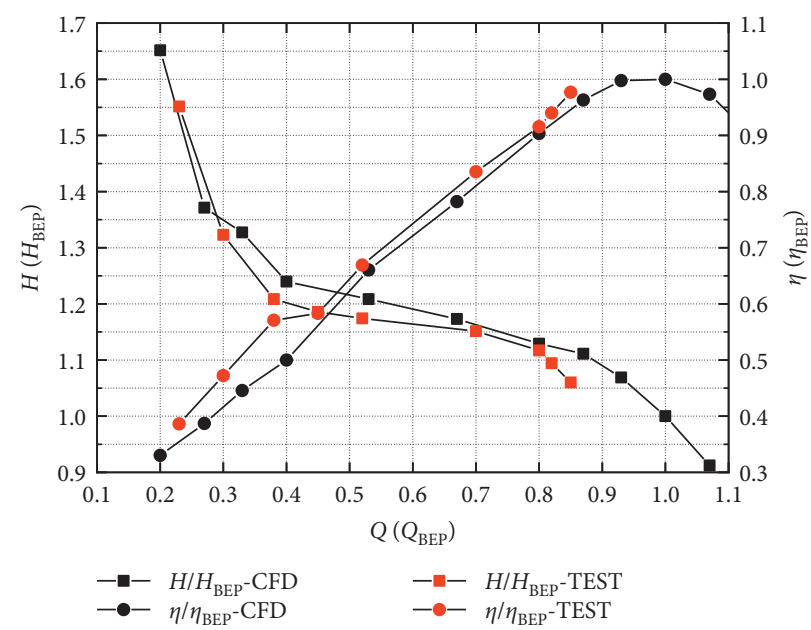

FIGURE 8: Efficiency of CFD and experimental results (BEP is an abbreviation for best-efficiency point. $\mathrm{Q}_{\mathrm{BEP}}$ is the discharge at the bestefficiency point. $\mathrm{H}_{\mathrm{BEP}}$ is the head at the best-efficiency point. $\eta_{\mathrm{BEP}}$ is the efficiency at the best-efficiency point.).

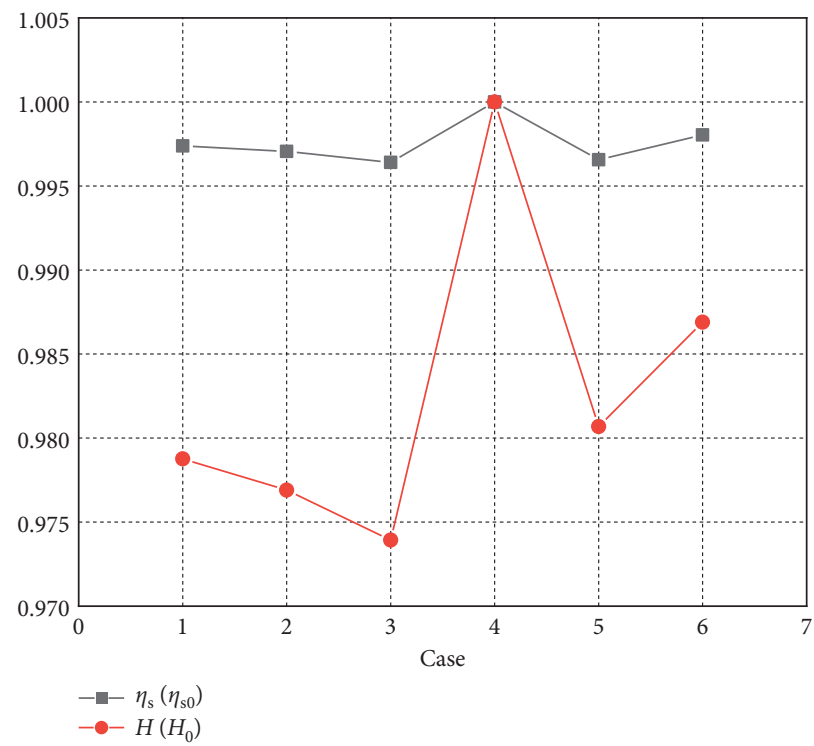

Figure 9: The law of head and system efficiency changing with the length of inlet duct.

range is within $0.5 \%$, indicating that the entire water jet propulsion pump device is little affected by the inlet duct length. The head of the water-jet propulsion pump varies greatly by the length of the inlet duct. The head of Case 3 is the lowest, which is reduced by $2.7 \%$, and the head of Case 4 is the highest. When the inlet duct is short (schemes 1,2, and 3 ), the net head is lower than that of scheme 4 due to the poor flow state of the inlet flow passage; when the flow passage is long (schemes 5 and 6), the net head will also be low due to the backflow. Overall, the runner's length of Case 4 is the most suitable.

Figure 10 shows the inlet duct efficiency with the length of the inlet duct. $\eta_{\mathrm{do}}$ is the inlet duct efficiency of the original scheme. It can be seen from the figure that the efficiency of the inlet duct increases first and then decreases as the length of the flow channel increases. Case 6 has the lowest efficiency of the inlet runner, which is reduced by $3.1 \%$. Although the channel is short (schemes 1,2, and 3), the loss along the channel is low, but the flow pattern is not good, which consumes a lot of energy, so the channel efficiency is not high; when the channel is long (schemes 5 and 6), the channel efficiency is also low because of the increase of the loss along the channel and the backflow loss. Therefore, Case 4 has the highest efficiency of the inlet duct, which is consistent with the original efficiency.

\subsection{Analysis of Flow Uniformity at the Outlet of the Inlet Duct.} Figure 11 is a cloud diagram of the velocity distribution of the inlet pipe and the length of the inlet pipe. The results show that there is a low-speed zone above the pump shaft and a high-speed zone below the pump shaft. As the length 


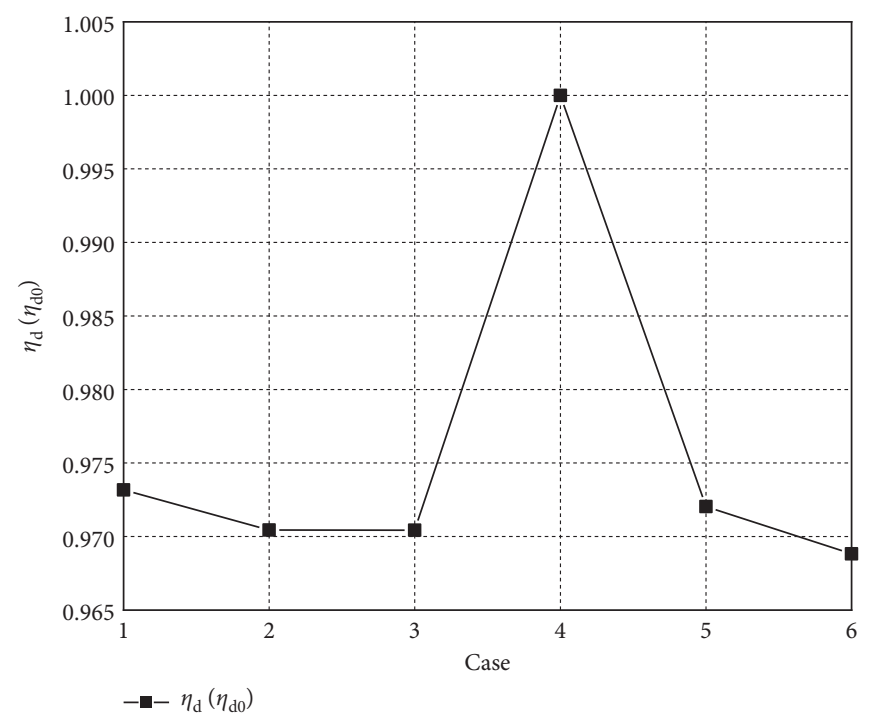

Figure 10: The inlet duct efficiency changes with the length of the inlet duct.

of the intake pipe increases, the area of the low-speed area above the drive shaft continuously decreases to almost zero, and the area of the high-speed area below the pump shaft also decreases to the middle, so the medium-speed distribution area continues to increase. This shows that as the length of the intake pipe increases, the velocity distribution moves closer to the middle value, the velocity gradient decreases, and the velocity uniformity of the entire water area increases.

Figure 12 is a graph showing the variation of the vorticity at the exit of the inlet duct with the length of the inlet duct. The figure shows that when the length of the inlet duct is short, the vorticity distribution in the area below the pump shaft is symmetrical on both sides. There is a negative vortex in the upper left area of the pump shaft. As the length of the inlet duct increases, the negative vortex area gradually decreases, and the absolute value of the vortex decreases, indicating that the strength of the vortex decreases with the increase of the inlet duct length. The positive vortex is distributed on the upper right side of the pump shaft. The closer to the upper side, the greater the vortex intensity. In particular, the vortex intensity at the upper right of Case 4 increases sharply, but the overall trend is that the vortex value decreases with the increase in the length of the inlet duct. Taken together, the vortex intensity of the outlet section of the inlet duct decreases with the length of the inlet duct.

Table 3 shows the velocity distribution uniformity $V_{u}$ and the weighted average angle $\theta$ of the outlet section of the inlet duct change with the inlet duct length. It can be seen from the table that the uniformity of velocity distribution is the largest when the length of the inlet duct is $1.2 \mathrm{~L}$, reaching $65.66 \%$, and the uniformity of velocity distribution is the smallest when the length of the inlet duct is $0.4 L$, indicating that the proper extension of the inlet duct length can improve the uniformity of the velocity distribution degrees, but the weighted average angle has little effect on the length of the inlet duct.
4.3. Analysis of Flow Characteristics in the Inlet Duct. Figure 13 is a static pressure streamline diagram of the section of the inlet duct with different inlet duct lengths. The figure shows that as the length of the inlet duct increases, the static pressure at the slope surface, inlet elbow, and lip corner of the inlet duct changes significantly. Specifically, the high-pressure area at the corner of the lip gradually decreases, and the static pressure at the slope surface and the inlet elbow does not change much.

The vortex inside the inlet duct occurs in the area above the inlet duct close to the drive shaft, which shows that the drive shaft hinders the flow of water. After Case 4, the vortex area obviously moves to the direction of the water inlet of the flow channel and leaves the vicinity of the drive shaft. This shows that when the length of the inlet duct increases to a certain extent, because the return area gradually increases, it is difficult for the propulsion pump device of the same speed to drive the water flow in the inlet flow passage into the pump body, so the fourth option is the best.

4.4. Thrust Characteristics. Figure 14 shows the change rule of thrust along with the extension of the inclined straight pipe section of the inlet duct. $F_{\mathrm{t} 0}$ is the thrust of the original scheme. With the extension of the inlet duct, the thrust of the entire device first increases and then decreases. The thrust of the fourth solution is the peak value. When the water inlet channel is shortened by 0.2 times or extended by 0.2 times, the thrust is reduced by $2.0 \%$, indicating that a certain length of the inlet channel is beneficial to the propulsion of the ship by the water jet propulsion pump device. The thrust depends on the exit speed. When the flow passage is short (schemes 1, 2 , and 3), the inlet velocity of the impeller chamber decreases due to the vortex and other bad flow patterns, so the outlet velocity of the shorter flow passage is smaller under the same speed of the impeller; when the flow passage is long (schemes 5 and 6), the increase of the loss along the way and the occurrence of the backflow phenomenon also leads to the 

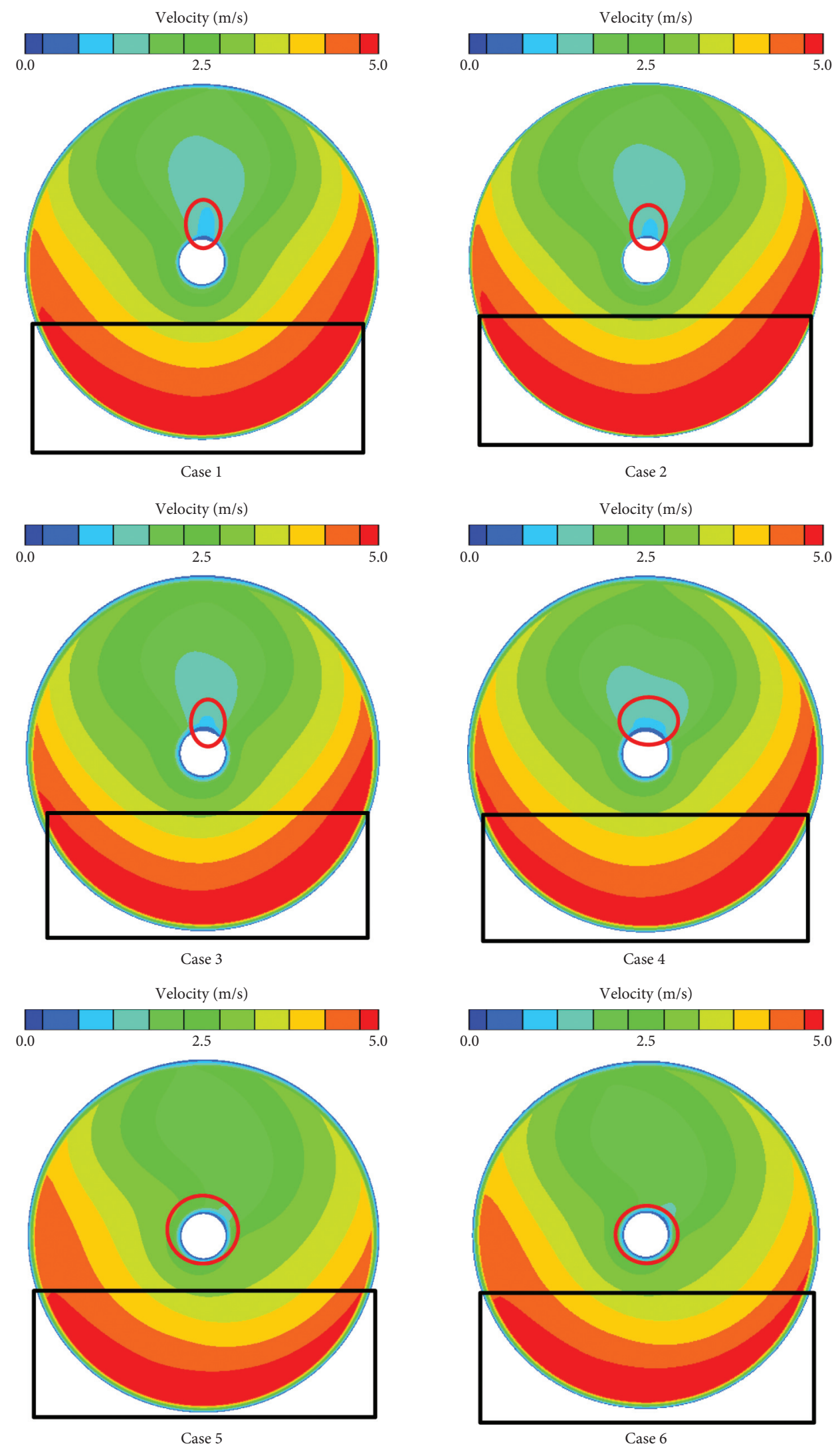

FIGURE 11: Cloud diagram of the velocity distribution at the exit of different inlet duct lengths. 

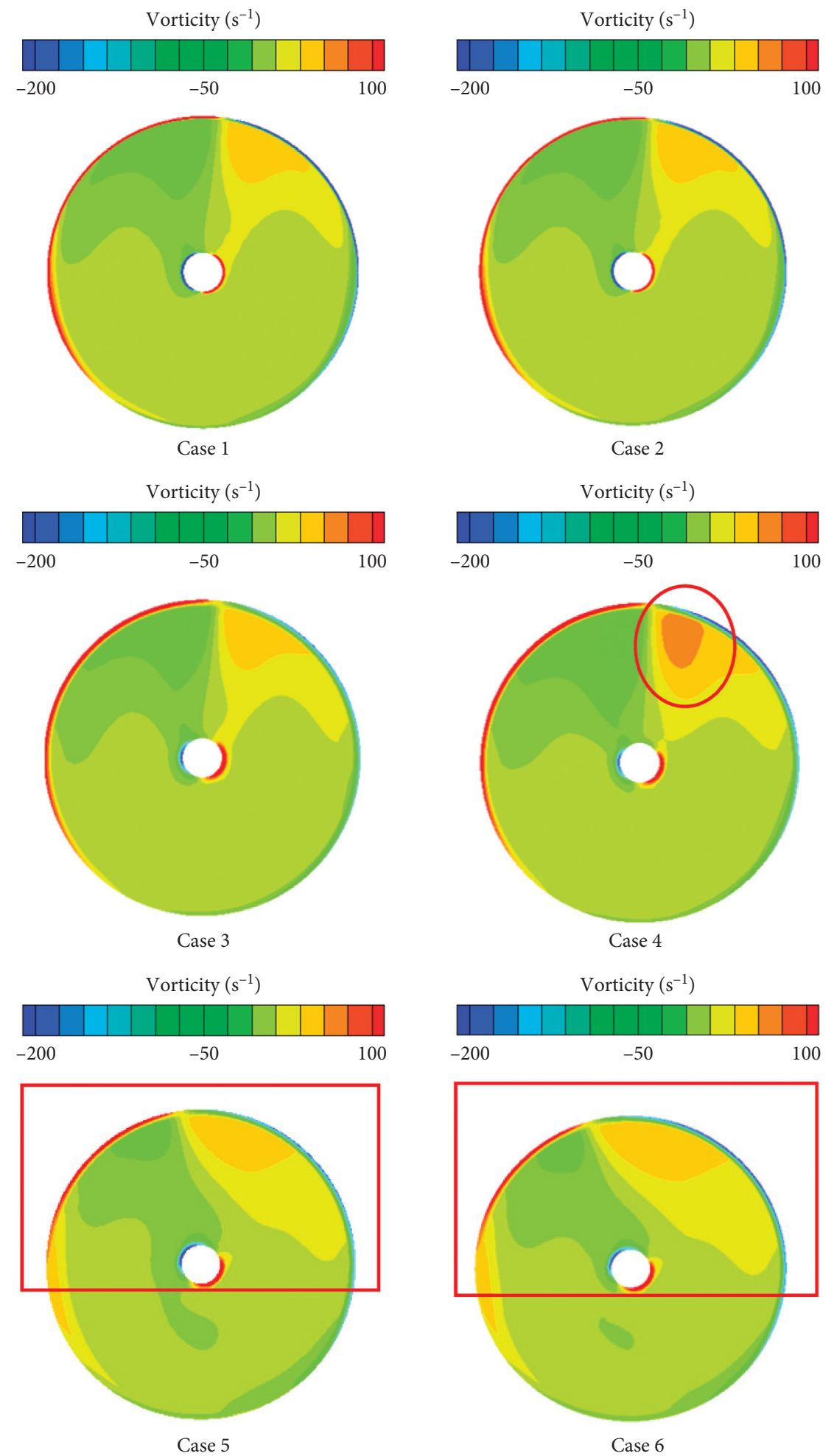

FIGURE 12: Cloud diagram of vorticity distribution at the exit of the runner with different runner lengths. 
TAвLE 3: The uniformity of velocity distribution $V_{u}$ and the weighted average angle $\theta$ vary with the length of the inlet duct.

\begin{tabular}{lcc}
\hline Case & Speed distribution uniformity $V_{u}(\%)$ & Weighted average angle $\theta\left(^{\circ}\right)$ \\
\hline $0.4 L$ & 60.09 & 77.97 \\
$0.6 L$ & 60.04 & 77.8 \\
$0.8 L$ & 60.22 & 78.81 \\
$1.0 L$ & 61.33 & 78.84 \\
$1.2 L$ & 65.66 & 78.61 \\
$1.4 L$ & 64.81 & 78.65 \\
\hline
\end{tabular}

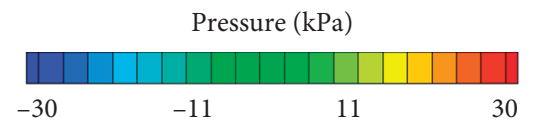

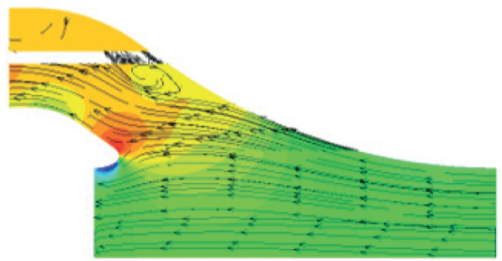

Case 1

Pressure $(\mathrm{kPa})$
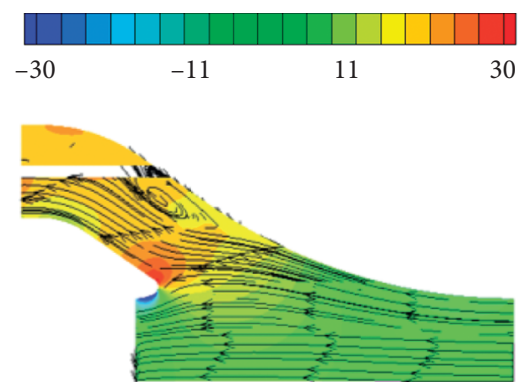

Case 3

Pressure (kPa)
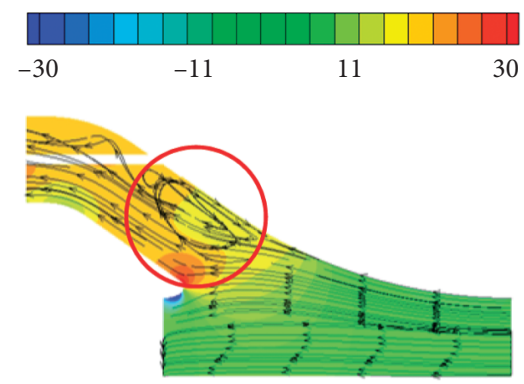

Case 5

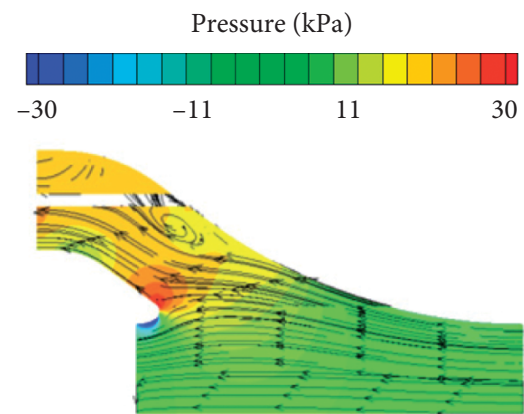

Case 2

Pressure $(\mathrm{kPa})$
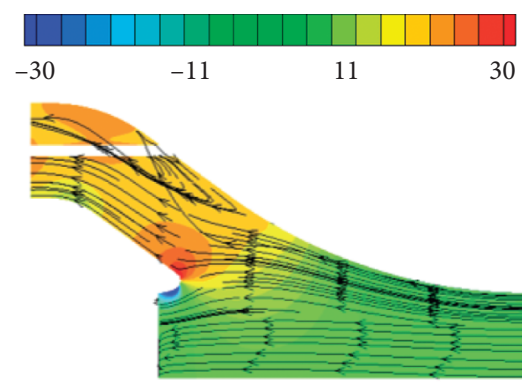

Case 4

Pressure (kPa)
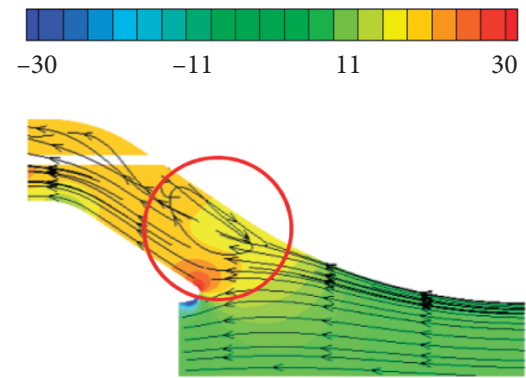

Case 6

Figure 13: The static pressure streamline diagram of the cross section in different inlet duct lengths. 


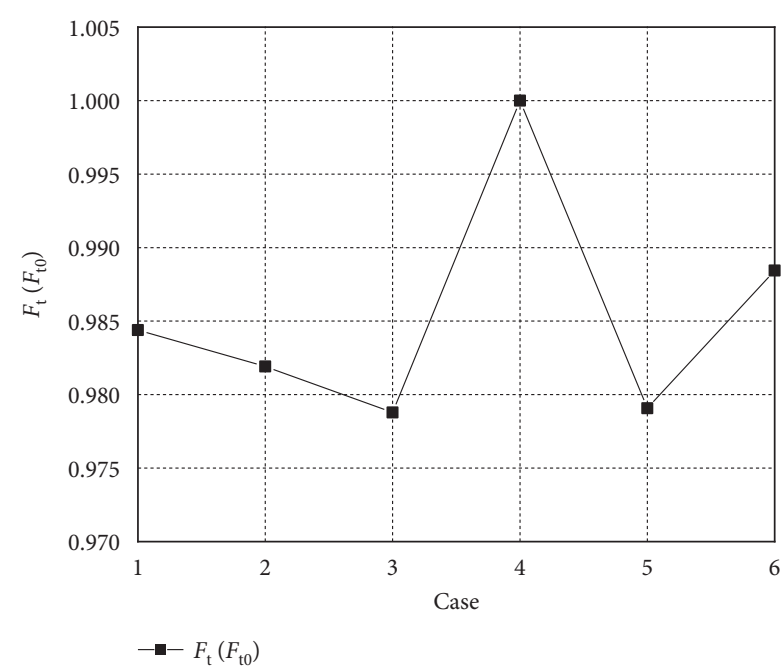

FIgURE 14: The thrust changes with the length of the inlet duct.

decrease of the inlet velocity of the impeller chamber. In this article, the thrust reaches the highest in Case 4.

\section{Conclusion}

Through a combination of numerical simulation and experimental verification, the influence of the change in the length of the inlet duct on itself and the entire water jet propulsion system is studied. The research conclusions are as follows:

(1) As the length of the inlet duct increases, both the head and the inlet duct efficiency firstly increase and then decrease. When $L=1.12 D$, they reached the peak value.

(2) Extending the length of the inlet duct is beneficial to improve the vortex condition and improve the uniformity of the water flow in the pipeline.

(3) The thrust firstly increases and then falls as the length of the inlet duct increases. When $L=1.12 D$, they reached the highest thrust value.

To sum up, among the six schemes studied in this article, scheme four has the highest comprehensive performance, and the length of the inclined straight pipe section is $L=1.12 D$.

\section{Data Availability}

The curve data used to support the findings of this study are available from the corresponding author upon request.

\section{Conflicts of Interest}

The authors declare that there are no conflicts of interest regarding the publication of this paper.

\section{References}

[1] T. Beek and H. Schooman, "Hybrid propulsion system," in Proceedings of the Advanced Naval Propulsion Symposium, Arlington, VA, USA, November 2004.

[2] C. Liu, Y. Wang, and J. Ding, "Overview of study of waterjet propulsion," Ship Engineering, vol. 4, pp. 57-60, 2006.

[3] X. He, Y. Zhang, C. Wang et al., "Influence of critical wall roughness on the performance of double-channel sewage pump," Energies, vol. 13, p. 464, 2020.

[4] H. Wang, B. Long, Y. Yang et al., "Modelling the influence of inlet angle change on the performance of submersible well pumps," International Journal of Simulation Modelling, vol. 19, no. 1, pp. 100-111, 2020.

[5] H. Wang, B. Long, C. Wang et al., "Effects of the impeller blade with a slot structure on the centrifugal pump performance," Energies, vol. 13, p. 1628, 2020.

[6] C. Wang, W. Shi, X. Wang et al., "Optimal design of multistage centrifugal pump based on the combined energy loss model and computational fluid dynamics," Applied Energy, vol. 187, pp. 10-26, 2017.

[7] L. Zhou, K. Deshpande, X. Zhang et al., "Process simulation of Chemical Looping Combustion using ASPEN plus for a mixture of biomass and coal with various oxygen carriers," Energy, vol. 195, Article ID 116955, 2020.

[8] L. Shi, W. Zhang, H. Jiao et al., "Numerical simulation and experimental study on the comparison of the hydraulic characteristics of an axial-flow pump and a full tubular pump," Renewable Energy, vol. 153, pp. 1455-1464, 2020.

[9] C. Wang, X. Chen, Q. Ning et al., "Numerical and experimental study on the pressure fluctuation, vibration, and noise of multistage pump with radial diffuser," Journal of the Brazilian Society of Mechanical Sciences and Engineering, vol. 40, no. 10, 2018.

[10] L. Zhou, W. Wang, J. Hang et al., "Numerical investigation of a high-speed electrical submersible pump with different end clearances," Water, vol. 12, p. 1116, 2020.

[11] L. Zhou, C. Han, L. Bai et al., "Numerical and experimental study of multiphase transient core-annular flow patterns in a spouted bed," ASME Journal of Energy Resource Technology, vol. 142, no. 9, Article ID 092104, 2020.

[12] D. Zhang, W. Jiao, L. Cheng et al., "Experimental study on the evolution process of the roof-attached vortex of the closed sump," Renewable Energy, vol. 164, pp. 1029-1038, 2021.

[13] H. Wang, Z. Qian, D. Zhang et al., "Numerical study of the normal impinging water jet at different impinging height, based on Wray-Agarwal turbulence model," Energies, vol. 13, p. $1744,2020$.

[14] H. Wang, Q. Hu, Y. Yang et al., "Performance differences of electrical submersible pump under variable speed schemes," International Journal of Simulation Modelling, vol. 20, no. 1, pp. 76-86, 2021.

[15] J. Zhou, M. Zhao, C. Wang et al., "Influence of different lateral bending angles on the flow pattern of pumping station lateral inflow," Shock and Vibration, vol. 2021, Article ID 6653001, 9 pages, 2021.

[16] J. Zhou, M. Zhao, C. Wang et al., "Optimal design of diversion piers of lateral intake pumping station based on orthogonal test," Shock and Vibration, vol. 2021, Article ID 6616456, 9 pages, 2021.

[17] W. Jiao, L. Cheng, D. Zhang et al., "Optimal design of inlet passage for waterjet propulsion system based on flow and 
geometric parameters," Advances in Materials Science and Engineering, vol. 2019, Article ID 2320981, 21 pages, 2019.

[18] W. Jiao, L. Cheng, D. Zhang et al., "Investigation of key parameters for the hydraulic optimization of the inlet duct based on a whole waterjet propulsion pump system," Transactions of Famena, vol. 45, no. 1, pp. 1-18, 2021.

[19] R. Huang, Y. Dai, X. Luo et al., "Multi-objective optimization of the flush-type intake duct for a waterjet propulsion system," Ocean Engineering, vol. 187, no. 1, pp. 106172.1-106172.14, 2019.

[20] Z. Gong, C. Song, G. Li et al., "Model predictive control for steering system of water-jet propulsion," Journal of Shanghai Jiaotong University (Science), vol. 25, no. 6, 2019.

[21] W. Park, H. Yun, H. Chun et al., "Numerical flow simulation of flush type inlet duct of waterjet," Ocean Engineering, vol. 32, no. 17-18, pp. 2107-2120, 2005.

[22] J. Ding and Y. Wang, "Research on flow loss of inlet duct of marine waterjets," Journal of Shanghai Jiaotong University (Science), vol. 15, no. 2, pp. 158-162, 2010.

[23] S. Xu, X. P. Long, B. Ji et al., "Vortex dynamic characteristics of unsteady tip clearance cavitation in a waterjet pump determined with different vortex identification methods," Journal of Mechanical Science and Technology, vol. 33, no. 12, pp. 5901-5912, 2019.

[24] P. Cao, Y. Wang, C. Kang et al., "Investigation of the role of non-uniform suction flow in the performance of water-jet pump," Ocean Engineering, vol. 140, pp. 258-269, 2017.

[25] R. Huang and X. Luo, "Numerical investigation of an intake duct for a waterjet propulsion system using modified partially averaged Navier-Stokes method," in Proceedings of the Fluids Engineering Division Summer Meeting, San Francisco, CA, USA, 2019.

[26] J. W. Lindau, W. L. Moody, M. P. Kinzel et al., Computation of Cavitating Flow through Marine Propulsors, Pennsylvania State University, State College, PA, USA, 2009.

[27] J. Gong, C. Guo, T. Wu et al., "Particle image velocimetry measurement of velocity distribution at inlet duct of waterjet self-propelled ship model," Journal of Hydrodynamics, vol. 29, no. 5, pp. 879-893, 2017.

[28] R. W. Kimball and T. E. Taylor, "Enhancements to the design and analysis of waterjet propulsors using a coupled liftingsurface/RANS technique including comparisons with experiment," in Proceedings of the 3rd International Conference of Waterjet Propulsion, The Royal Institution of Naval Architects (RINA), Gothenburg, Sweden, February 2001.

[29] J. E. Kerwin, "Hydrodynamic issues in waterjet design and analysis," in Proceedings of the 26th Symposium on Naval Hydrodynamics, Rome, Italy, September 2006.

[30] J. Gong, C. Guo, C. Wang et al., "Analysis of waterjet-hull interaction and its impact on the propulsion performance of a four-waterjet-propelled ship," Ocean Engineering, vol. 180, pp. 211-222, 2019.

[31] S. Chang, Y. Wang, Z. Pang et al., "Research on effects of IVR conditions in waterjet inlet performance," Journal of Wuhan University of Technology, vol. 34, no. 4, pp. 721-724+729, 2010.

[32] S. Lei, Study of Grid Error in CFD Simulation Based on Richardson Extrapolation Method, North China Electric Power University, Beijing, China, 2011.

[33] C. Wang, X. He, L. Cheng et al., "Numerical simulation on hydraulic characteristics of nozzle in waterjet propulsion system," Processes, vol. 7, no. 12, p. 915, 2019.

[34] P. Jin, Marine Water-Jet Propulsion, National Defense Industry Press, Beijing, China, 1986.
[35] C. Liu, Pump and Pumping Station, China Water \& Power Press, Beijing, China, 2009.

[36] F. Yang, X. Wang, J. Jiang et al., "Parametric analysis of the inlet duct in the marine waterjet propulsor," Journal of $\mathrm{Me}$ chanical \& Electrical Engineering, vol. 36, no. 11, pp. 12121215, 2019.

[37] S. Chang, Y. Wang, J. Ding et al., "Hydraulic design and performance predictions of a waterjet mixed-flow pump," Journal of Harbin Engineering University, vol. 32, no. 6, pp. 708-713, 2011.

[38] C. Xia, L. Cheng, C. Luo et al., "Hydraulic characteristics and measurement of rotating stall suppression in a waterjet propulsion system," Transactions of FAMENA, vol. 42, no. 4, pp. 85-100, 2019. 